Tohoku J. Exp. Med., 2008, 216, 149-156

\title{
Differentiation of Adipose-derived Stromal Vascular Fraction Culture Cells into Chondrocytes Using the Method of Cell Sorting with a Mesenchymal Stem Cell Marker
}

\author{
Daisuke Ishimura, ${ }^{1}$ NaOKi Yamamoto, ${ }^{2}$ Kaori Tajima, ${ }^{1}$ Ayumi Ohno, ${ }^{1}$ \\ Yasuhiro Yamamoto, ${ }^{1}$ Osuke Washimi ${ }^{1}$ and Harumoto Yamada ${ }^{1}$ \\ ${ }^{1}$ Department of Orthopaedic Surgery, School of Medicine, Fujita Health University, Aichi, Japan \\ ${ }^{2}$ Laboratory of Molecular Biology and Histochemistry, Joint Research Laboratory, Fujita Health \\ University, Aichi, Japan
}

The incidence of arthritic diseases is rapidly increasing in most advanced countries. Articular cartilage, which is the most important tissue in the joint, consists of chondrocytes and abundant extracellular matrix, including aggrecan, and shows poor self-repair. We studied the potential of stem cells in mouse subcutaneous adipose tissue as a source of cells to regenerate cartilage tissue. Analysis of adipose-derived stromal vascular fraction culture cells (ADSVFs) using mesenchymal stem cell markers showed that CD90-positive cells accounted for 93.8\%, CD105-positive cells for $68.5 \%$, and 75 neurotrophin receptor (p75NTR, CD271)-positive cells for $36.1 \%$. These results indicate that cells positive for mesenchymal stem cell markers are present in ADSVFs. The CD105-positive or -negative cells were isolated from ADSVFs by magnetic cell separation (MACS), and the efficiency of differentiation into chondrocytes was compared with using three methods of pellet method, gel-coating method, and gel-embedding sheet method. Using the CD105-positive cells and the gel-embedding sheet method, aggrecan mRNA was detected about three times higher than pellet and gel-coating methods. The above data suggest that ADSVFs could be differentiated into chondrocyte-like cells in the gel-embedding sheet method and could be useful in regenerative medicine to treat cartilage defects or cartilage degenerative disease. The use of cells sorted by mesenchymal stem cell markers from adipose tissue would gain position in the repair of cartilage tissue. chondrocyte; adipose-derived stromal vascular fraction culture cells (ADSVFs); CD105; mesenchymal stem cell; gel-embedding sheet.

Tohoku J. Exp. Med., 2008, 216 (2), 149-156.

(C) 2008 Tohoku University Medical Press

Articular cartilage is hyaline cartilage consisting of chondrocytes and abundant extracellular matrix (mainly type II collagen and aggrecan).
Blood vessels, nerves, and lymph are absent in cartilage tissues, and the movement of chondrocytes is markedly inhibited by the surrounding

Received June 25, 2008; revision accepted for publication August 28, 2008.

Correspondence: Naoki Yamamoto, Laboratory of Molecular Biology and Histochemistry, Joint Research Laboratory, Fujita Health University, 1-98 Dengakugakubo, Kutsukake-cho, Toyoake-city, Aichi 470-1192, Japan. e-mail: naokiy@fujita-hu.ac.jp 
extracellular matrix. Thus, the mechanism for healing and self-repair, including cell mobilization and infiltration, is unlikely to occur, even when articular cartilage is impaired or degenerated. To repair articular cartilage injuries, various treatment methods have been attempted, such as a drilling method (Mitchell et al. 1976), which is a bone marrow stimulation method, a microfracture method (Stedman et al. 2001), periosteal/ perichondrial transplantation (O'Driscoll et al. 1986), and mosaic plasty (Matsusue et al. 1993), but no method has been established. Regenerative medicine for cartilage tissue repair utilizing cell culture techniques has recently been investigated worldwide: transplantation of cultured autologous chondrocytes (Brittberg et al. 1994; Wakitani et al. 1994; Ochi et al. 2002), and chondrocytes differentiated from tissues other than cartilage, such as bone marrow, synovial cells, and adipocytes; however, problems remain to be resolved: it requires two separate surgeries for cell sampling and transplantation, and long-term maintenance of the characteristics of differentiated chondrocytes is difficult. For these reasons, repair with complete hyaline cartilage has not yet been confirmed (Benya et al. 1982; Sohn et al. 2002).

As a basic study on regenerative medicine of cartilage tissue aiming at future clinical applications, we focused on tissue stem cells (mesenchymal stem cells) present in adipose tissue as cell supply sources other than chondrocytes because they have high proliferative ability and the potential to differentiate into various cell types, such as adipocytes, osteoblasts, chondrocytes, myofibroblasts, and nerve cells (Caplan 1991; Pittenger et al. 1999; Yamamoto et al. 2007). In this study, we isolated adipose-derived stromal vascular fraction culture cells (ADSVFs) using mesenchymal stem cell markers, and investigated the differentiation of highly pure adipose-derived stem cells (ASCs) with no contaminating vascular endothelial cells or fibroblasts into chondrocytes (Halvorsen et al. 2001; Kino-oka et al. 2005; Xu et al. 2007).

\section{Material And Methods}

Cell isolation and culture of adipose tissue

Cells were isolated from lymph node-excised ingui- nal subcutaneous adipose tissue of ICR mice (4 weeks of age, male, 18-20 g). Briefly, after collagenase treatment, hemolysis, and filtration of adipose tissue, the sample was washed with phosphate-buffered saline (PBS: Sigma, St. Louis, MO) by centrifugation. The cell pellet was cultured in Dulbecco's modified Eagle's medium/ Ham's F12 (DMEM/F12, Sigma) containing 10\% fetal bovine serum (FBS, JRH Biosciences, Adelaide, Australia), $2 \mathrm{ng} / \mathrm{ml}$ basic fibroblast growth factor (bFGF, Sigma), and $1 \%$ penicillin-streptomycin (Sigma) at $37^{\circ} \mathrm{C}$ in 5\% $\mathrm{CO}_{2}$. After culture for 24 hours, cells adhered to the culture dish were further cultured as ADSVFs for 6 days. All procedures were approved by the Education and Research Center for Animal Models of Human Diseases of Fujita Health University.

\section{Immunohistological staining of adipose tissue}

ICR mouse subcutaneous adipose tissue, including inguinal skin tissue, was excised and rapidly fixed with a rapid tissue fixative (Super Fix KY-500; KURABO, Osaka Japan), which rapidly fixes even adipose and eyeball tissues, and paraffin-embedded sections were prepared using the standard method (Yamamoto et al. 2008). Sections were reacted with the following primary antibodies of mesenchymal stem cell markers: rat monoclonal CD90 and CD105 (Thy-1 and Endoglin, respectively; eBioscience, Inc., San Diego, CA) antibodies and rabbit polyclonal p75 neurotrophin receptor (p75NTR, CD271; Chemicon International, Inc., Temecula, CA) antibody. After washing with PBS, the sections were reacted with Alexa Fluor ${ }^{\mathbb{B}} 488$ (Invitrogen Corp., Carlsbad, CA) as the secondary antibody. DAPI (Vectashield H-1200, Vector Laboratories, Burlingame, CA, USA) was used for nuclear staining. The sections were then observed under a microscope (Power BX-51; Olympus, Tokyo, Japan).

\section{Flow cytometric analysis}

Cells immediately isolated from adipose tissue or after 7 days culture (ADSVFs) were subjected to cell surface marker analysis using a cell analyzer (fluorescence-activated cell sorting: FACS, FACSCan, BD Biosciences San Jose, CA). The cells were reacted with primary antibodies of CD11b (Integrin- $\alpha \mathrm{M}$ ), CD29 (Integrin- $\beta 1$, Chemicon), CD31 (PECAM-1), CD34, CD45 (leukocyte common antigen), CD90, CD105, CD106 (VCAM, Santa Cruz Biotechnology, Inc., Santa Cruz, CA), CD117 (c-Kit), CD133 (Prominin-1) (all were purchased from eBioscience, excluding CD29, 
CD106), p75NTR (CD271, Chemicon), and Sca-1 (Stem cell antigen-1 /Ly6, R\&D Systems, Inc., Minneapolis, $\mathrm{MN})$. After reacting with antibodies at $4^{\circ} \mathrm{C}$ for $30 \mathrm{~min}$ utes, the cells were washed with PBS, followed by a reaction with the secondary antibody, Alexa Fluor ${ }^{\circledR} 488$, at $4^{\circ} \mathrm{C}$ for 30 minutes. After washing with PBS, the cells were analyzed by FACS. These experiments were repeated 3 times.

\section{Cell density in differentiation culture}

The initial cell density for differentiation into chondrocytes in culture was investigated. After ADSVFs were cultured for 7 days, cell density was adjusted to $1 \times$ $10^{5}, 2 \times 10^{5}, 5 \times 10^{5}$, and $10 \times 10^{5}$ cells $/ \mathrm{ml}$. Differentiation into chondrocytes was induced by cell centrifugation and pellet culture (pellet method) in chondrocyte differentiation medium (CA411D250, TOYOBO, Osaka, Japan) for 20 days, and the influence of cell density on differentiation was investigated.

\section{Magnetic cell separation}

ADSVFs were labeled with rat anti-CD105 monoclonal antibody, and reacted with microbead-labeled anti-rat IgG antibody (Miltenyi Biotec $\mathrm{GmbH}$, Bergisch Gladbach, Germany). The cells were then separated into CD105-positive or -negative cells by a magnetic-activated cell sorting (MACS, Miltenyi Biotec $\mathrm{GmbH}$ ) method using a magnetic column.

\section{Cell differentiation into chondrocytes}

CD105-positive and -negative cells separated by MACS were adjusted to the cell density of $5 \times 10^{5}$ cells/ $\mathrm{ml}$, and subjected to pre-differentiation culture in DMEM/F12 medium containing 10\% FBS and $2 \mathrm{ng} / \mathrm{ml}$ bFGF for 5 days. Cell density was then adjusted to $2 \times$ $10^{5}$ cells $/ \mathrm{ml}$, and the cells were cultured in the presence of $8 \%$ FBS and $2 \mathrm{ng} / \mathrm{ml} \mathrm{bFGF}$ for 2 days, followed by medium change to chondrocyte differentiation medium (CA411D250), and the cells were cultured for 20 days. Control cells were cultured in DMEM/F12 medium containing FBS and bFGF for the same period.

In addition to the typical chondrocyte differentiation method, the pellet culture method, involving culture by seeding cells on atelocollagen gel (KOKEN, Tokyo Japan) (gel-coating method), in which chondrocytes proliferate while maintaining their characteristics, and embedding cells in a gel sheet (gel-embedding sheet method) were also performed as alternative culture conditions for differentiation, and total RNA was extract- ed from cells cultured for 20 days (High Pure RNA Isolation Kit, Roche Diagnostics Corp., Indianapolis, IN). Total RNA was subjected to quantitative real-time RT-PCR (qRT-PCR) (PRISM-7900 HT, Applied Biosystems Inc., Foster City, CA) to measure the expressions of chondrocyte differentiation markers, the Runtrelated gene 2 (Runx2, Mm03003491_m1, ABI) and mature chondrocyte differentiation markers, aggrecan (Mm00545794_m1, ABI), and housekeeping geneencoded glyceraldehyde-3-phosphate dehydrogenase (GAPDH: Mm99999915_g1), for comparison. ADSVFs not separated based on CD105 were cultured in serumsupplemented medium without medium change to differentiation medium for the same period, and the mRNA expression level was regarded as the baseline.

\section{Immunostaining of differentiation cells}

After 20-day differentiation into chondrocytes, the cells were fixed with $4 \%$ paraformaldehyde at $4{ }^{\circ} \mathrm{C}$ for 30 minutes. Frozen sections were prepared from cells cultured by the pellet and gel-coating methods using a cryostat (CM3050S Leica Microsystems, Nussloch, Germany), and stained. Cells cultured by the gel-embedded method were stained in the gel. After reacting with anti-aggrecan antibody (Abcan Limited, Cambridgeshire, UK) at $37^{\circ} \mathrm{C}$ for 1 hour, the preparations were washed 3 times with PBS, reacted with the secondary antibody of Alexa Fluor ${ }^{\circledR} 488$ at $37^{\circ} \mathrm{C}$ for 1 hour, and washed 3 times with PBS followed by nuclear staining with DAPI (Vectashield H-1200).

\section{Statistical Analysis}

Data are presented as the means \pm S.D. The significance of differences between two groups was evaluated using ANOVA. The $p$ value $<0.05$ was considered significant.

\section{RESULTS}

\section{Immunostaining of subcutaneous adipose} tissue

Mouse adipose tissue from the subcutaneous inguinal region was used. Many CD90 (Fig. 1A), CD105 (Fig. 1B), and p75NTR (Fig. 1C) -positive cells were found to be distributed around microblood vessels in the deeper layers of adipose tissue. Positive cells were comparatively small, within a narrow range. 

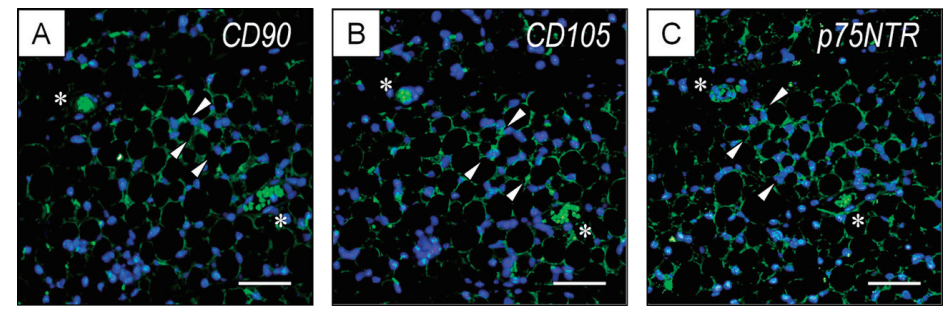

Fig. 1. Immunohistological staining of mouse subcutaneous adipose tissue.

Staining of CD90 (A), CD105 (B), p75NTR (C). Cells positive for each marker (arrowheads) were present around the micro-blood vessels $(*)$ in deep adipose tissue. $\mathrm{Bar}=50 \mu \mathrm{m}$.

\section{Characterization of ADSVFs}

Since lymph nodes present in adipose tissue can be easily distinguished, they were dissected in advance. CD90-positive cells accounted for 49.3\%, CD105-positive cells for $47.0 \%$, and p75NTR-positive cells for $23.6 \%$ after immediately isolation from adipose tissue, but the rates increased to $93.8 \%, 68.5 \%$, and $36.1 \%$ after 7-day culture (ADSVFs), respectively. In contrast, CD34, known as a hematopoietic stem cell and vascular endothelial precursor cell marker, CD45 as a hematopoietic cell marker, and CD31 as a vascular endothelial cell marker decreased (Table 1). We increased the positive cell rate of the mesenchymal stem cell marker by adding appropriate serum concentration and growth factor to the culture. From these results, we proved that positive cells of the mesenchymal stem cell marker could be increased even from a small cell count or little tissue.

\section{Cell density at culture differentiation into chon- drocyte}

The influence of cell density on ADSVF differentiation into chondrocytes was evaluated from the chondrocyte marker of aggrecan by qRT-PCR. The aggrecan mRNA level was less than the detection limit when the initial cell density was $1 \times 10^{5}$ cells $/ \mathrm{ml}$, but it was detected when the differentiation density was $2 \times 10^{5}$ cells $/ \mathrm{ml}$ or higher. This result proved that cell density was very important for differentiation into chondrocytes.

Separation of CD105-expressing cells

ADSVFs after 7-day culture were analyzed
TABLE 1 Flow cytometric analysis of ADSVF cells.

\begin{tabular}{lrrc}
\hline & \multicolumn{2}{c}{ Mean pnnitiae cell markern $( \pm$ S.D. $)$} \\
\cline { 2 - 2 } in vivo cells & & primary cullure cells \\
\hline CD11 & $9.2 \pm 0.5$ & $0.3 \pm 0.1$ \\
CD29 & $70.4 \pm 4.5$ & $94.5 \pm 4.3$ \\
CD31 & $2.2 \pm 0.4$ & $0.5 \pm 0.1$ \\
CD34 & $23.6 \pm 1.2$ & $4.6 \pm 2.4$ \\
CD45 & $0.97 \pm 0.1$ & $0.7 \pm 0.2$ \\
CD90 & $49.3 \pm 0.3$ & $93.8 \pm 3.3$ \\
CD105 & $47.0 \pm 1.5$ & $68.5 \pm 2.9$ \\
CD106 & $7.2 \pm 2.3$ & $0.9 \pm 0.7$ \\
CD117 & $1.9 \pm 0.1$ & $0.6 \pm 0.3$ \\
CD133 & $2.0 \pm 0.3$ & $0.4 \pm 0.2$ \\
p75NTR & $23.6 \pm 6.8$ & $36.1 \pm 5.9$ \\
Sca-1 & $5.8 \pm 1.7$ & $94.7 \pm 0.5$ \\
\hline
\end{tabular}

with regard to a mesenchymal stem cell marker, CD105 (Fig. 2A). CD105-positive and -negative cells showed an apparent bimodal distribution (Fig. 2B), and could be separated by MACS using CD105 (Fig. 2C, D). This result proved that the intended or expected cells were separated using the MACS method the same as with a cell sorter. Comparing MACS with a cell sorter, all steps of the MACS method could be performed on a clean bench, and did not require expensive research equipment or extensive technology.

Compared with cell differentiation into chondrocytes

CD105-positive or -negative cells separated by MACS were subjected to 20-day differentiation under three culture conditions, in all of which, aggrecan and Runx 2 mRNA expression 

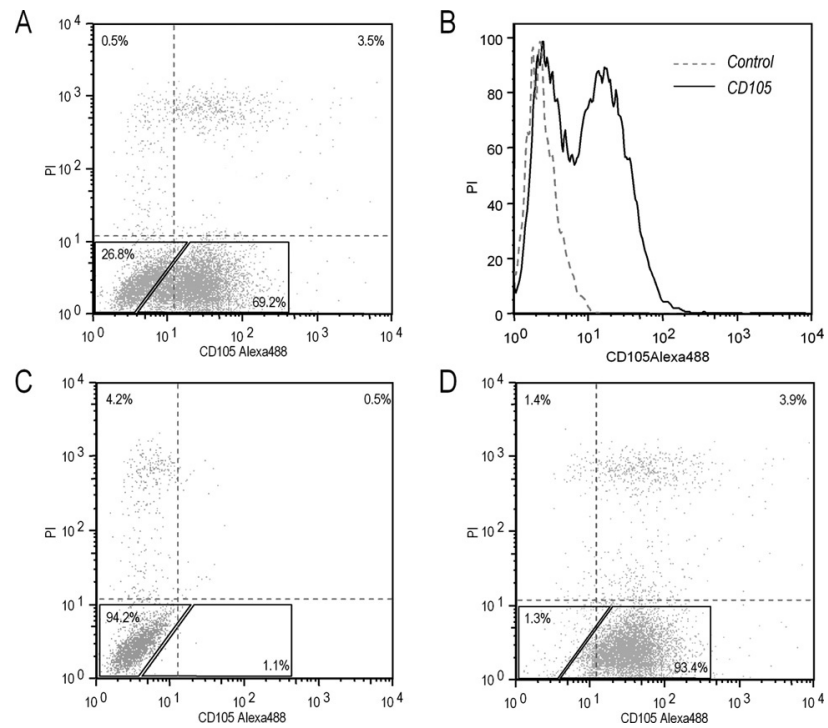

Fig. 2. Separation of ADSVFs by CD105.

Cells were separated into CD105-positive or -negative cells by MACS. Before MACS separation of ADSVFs (A), histogram of the same sample (B), after MACS separation of CD105-negative cells (C) and CD105-positive cells (D) achieved over $90 \%$ purity.
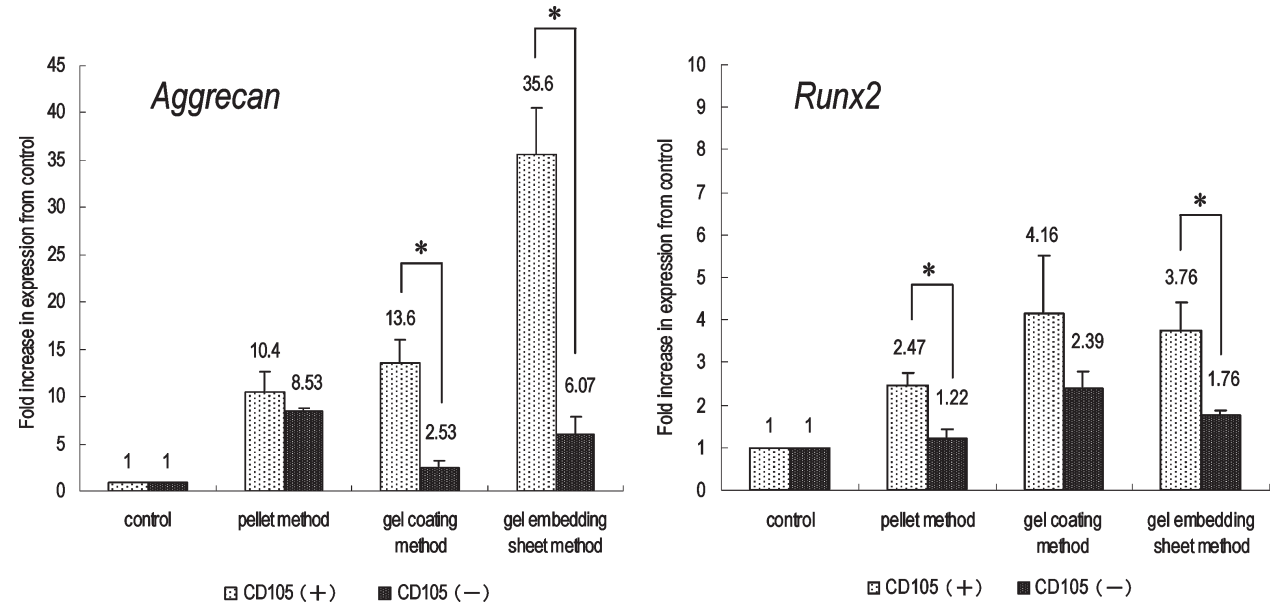

Fig. 3. Investigation of culture conditions using CD105-positive and -negative cells.

The highest aggrecan mRNA expression level was detected in the gel-embedding method using CD105-positive cells, in comparison with the control. The Runx 2 mRNA expression level showed the higher detection of differentiation cells from CD105-positive cells than CD105-negative cells $(*: p<0.05$, ANOVA).

levels were higher the differentiation cells from CD105-positive cells than CD105-negative cells. Regarding the culture conditions, aggrecan mRNA of mature chondrocyte differentiation markers was significantly different between CD105-positive and -negative cells cultured by the gel-embedding sheet method (ANOVA). Runx2 mRNA therefore showed higher detection of differentiation cells from CD105-positive cells than CD105-negative cells (ANOVA) (Fig. 3). We supposed that the gel-embedding sheet method could culture chondrocytes with high cell den- 

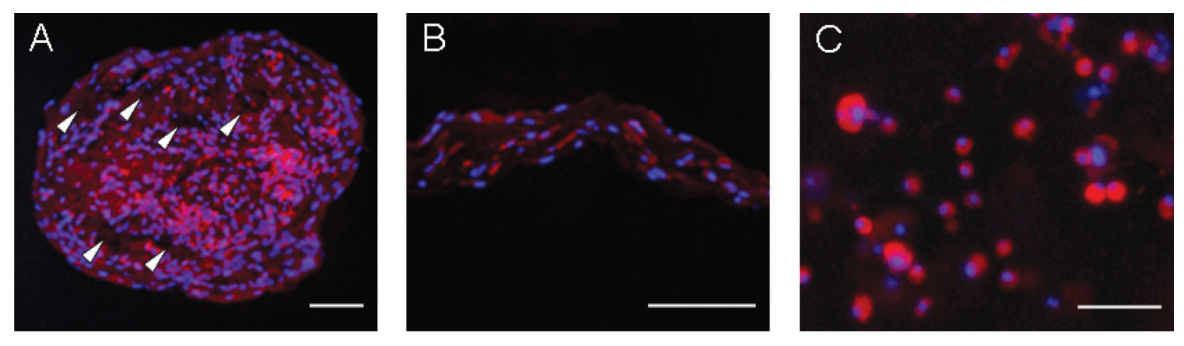

Fig. 4. Immunostaining of aggrecan in differentiated cells.

The region of defective cells (arrowheads) was observed in cell aggregation as pellets by the pellet method (A). Cultured cells of the gel-coating method formed multiple layers, and a different expression of aggrecan was observed by region (B). The cultured cells of the gel-embedding method showed a spherical form (C). Bar $=100 \mu \mathrm{m}$.

sity from three dimensions, and increased the expression level of aggrecan mRNA. We therefore considered that the gel-embedding sheet method was the most suitable geometrically to regenerative cartilage tissue.

\section{Aggrecan immunostaining of differentiation cells}

When cells were differentiated from chondrocytes by pellet (Fig. 4A), gel-coating (Fig. 4B), and gel-embedding sheet (Fig. 4C) methods, aggrecan-positive cells were present in all cultures. The pellet method was used in a defective region, and the gel-coating method showed a different expression level of aggrecan by region. On the other hand, the cells of the gel-embedding sheet method were observed to be spherical, the same as in vivo cartilage tissue.

\section{DiscuSSION}

We consider that minimizing invasiveness and increasing the induction efficiency of a small number of cells into chondrocytes are most important in the regeneration of cartilage tissue. For this purpose, ADSVFs isolated and cultured from adipose tissue were sorted using mesenchymal stem cell markers to remove vascular endothelial cells and fibroblasts in ADSVFs (mesenchymal stem cell marker-negative cells). This unique procedure increased the efficiency of inducing differentiation into chondrocytes. To our knowledge, this is the first paper in which mesenchymal stem cell markers were utilized for cell sorting to increase the induction of differenti- ation into highly pure chondrocytes.

The induction of chondrocyte differentiation from ADSVFs was investigated using various cell densities at culture initiation by the pellet method. The mRNA of a chondrocyte marker, aggrecan, was detected when the initial cell density was $2 \times$ $10^{5}$ cells $/ \mathrm{ml}$ or higher, but the mRNA level was less than the detection limit when the initial density was $1 \times 10^{5}$ cells $/ \mathrm{ml}$ or lower, showing that cell density at the initiation of induction was important for differentiation into chondrocytes, and $2 \times 10^{5}$ cells $/ \mathrm{ml}$ or higher cell density was necessary for the differentiation of mouse ADSVFs into chondrocytes.

When CD105-positive cells were cultured by the gel-embedding sheet method, the aggrecan mRNA level in differentiated cells was higher than that in cells differentiated by the pellet culture method, suggesting that the isolation of cells from ADSVFs using a mesenchymal stem cell marker, CD105, increased the purity of adipose stem cells (ASCs), which realized the objective of this study: "increasing differentiation into chondrocytes using a minimum number of cells'. Since CD105 expression was maintained after culture passages (Yoshimura et al. 2006), it is an attractive marker in this field.

In cultures by the pellet method, small tear drop-like aggregates are formed. Considering the low metabolic activity of cells in the center of aggregates and the difficulty of harvesting aggregates of the size of the cartilage defect in patients, clinical application of the pellet method may be difficult. In this point, the gel-embedding sheet 
method has many advantages. The present data suggest that this culture method may be more useful for articular cartilage injury in the combination with a cell-supporting system.

There have been many discussions about the selection of donor tissue for regenerative medicine. The reasons for selecting adipose tissue in the present study aiming at clinical application are as follows: firstly, ASCs have very high proliferative ability, with potential to differentiate into mesenchymal cells, such as adipocytes, osteoblasts, chondrocytes, and myofibroblasts (Zuk et al. 2001), similarly to bone marrow-derived mesenchymal stem cells: multipotent adult progenitor cells (MAPCs) (Jiang et al. 2002), and multipotency transcending the developmental framework, such as differentiation into nerve, vascular endothelial, and liver cells (Strem et al. 2005; Yamamoto et al. 2007). Another reason is that patients may easily accept the cell-sampling procedure for ASCs. Generally, adipose tissue accounts for about $20 \%$ of the body weight on average, and is considered merely as an energy store. Since patients can easily imagine adipose tissue sampling (adipocytes), compared to sampling bone marrow and synovial cells, and consider the tissue unnecessary, we expect that patients will readily understand and accept the cell-sampling procedure for regenerative medicine of cartilage tissue.

On the other hand, various problems still exist regarding the use of ASCs for the regeneration of cartilage tissue. The previous article reported that ASCs showed poorer osteogenic and chondrogenic potential than MAPCs (Im et al. 2005). It has also been reported that synovial tissue has greater potential to differentiate into chondrocytes than adipose tissue, suggesting a problem in selecting sampling regions (Sakaguchi et al. 2005; Wickham et al. 2003). Furthermore, possible carcinogenesis in ASCs in long-term culture (4-5 months) has been reported (Rubio et al. 2005) showing the necessity of reconfirming the safety of these cells.

However, as yet there has been no report in which mesenchymal stem cell markers were sorted, or the induction of differentiation into highly pure chondrocytes was investigated. We are now performing comparative studies with MAPCs to verify these problems. The present data indicated that ADSVFs could be highly differentiated into chondrocytes through cell sorting using mesenchymal stem cell markers and have advantages in regenerative medicine for the treatment of cartilage defects or cartilage degenerative disease.

\section{References}

Benya, P.D. \& Shaffer, J.D. (1982) Dedifferentiated chondrocytes reexpress the differentiated collagen phenotype when cultured in agarose gels. Cell, 30, 15-24.

Brittberg, M., Lindahl, A., Nilsson, A., Ohlsson, C., Isaksson, O. \& Peterson, L. (1994) Treatment of deep cartilage defects in the knee with autologous chondrocyte transplantation. N. Engl. J. Med., 331, 889-895.

Caplan, A.I. (1991) Mesenchymal stem cells. J. Orthop. Res., 9, 641-650.

Halvorsen, Y.D., Bond, A., Sen, A., Franklin, D.M., Lea-Currie, Y.R., Sujkowski, D., Ellis, P.N., Wilkinson, W.O. \& Gimble, J.M. (2001) Thiazolidinediones and glucocorticoids synergistically induce differentiation of human adipose tissue stromal cells: biochemical, cellular, and molecular analysis. Metabolism, 50, 407-413.

Im, G.I., Shin, Y.W. \& Lee, K.B. (2005) Do adipose tissuederived mesenchymal stem cells have the same osteogenic and chondrogenic potential as bone marrow-derived cells? Osteoarthritis and Cartilage, 13, 845-853.

Jiang, Y., Jahagirdar, B.N., Reinhardt, R.L., Schwartz, R.E., Keene, C.D., Ortiz-Gonzalez, X.R., Reyes, M., Lenvik, T., Lund, T., Blackstad, M., Du, J., Aldrich, S., Lisberg, A., Low, W.C., Largaespada, D.A. \& Verfaillie, C.M. (2002) Pluripotency of mesenchymal stem cells derived from adult marrow. Nature, 418, 41-49.

Kino-Oka, M., Maeda, Y., Yamamoto, T., Sugawara, K. \& Taya, M. (2005) A kinetic modeling of chondrocyte culture for manufacture of tissue-engineered cartilage. J. Biosci. Bioeng., 99, 197-207.

Matsusue, Y., Yamamuro, T. \& Hama, H. (1993) Arthroscopic multiple osteochondral transplantation to the chondral defect in the knee associated with anterior cruciate ligament disruption. Arthroscopy, 9, 318-321.

Mitchell, N. \& Shepard, N. (1976) The resurfacing of adult rabbit articular cartilage by multiple perforations through the subchondral bone. J. Bone Joint Surg. Am., 58, 230-233.

Ochi, M., Uchio, Y., Kawasaki, K., Wakitani, S. \& Iwasa, J. (2002) Transplantation of cartilage -like tissue made by tissue engineering in the treatment of cartilage defects of the knee. J. Bone Joint Sur. Br., 84, 571-578.

O'Driscoll, S.W. \& Salter, R.B. (1986) The repair of major osteochondral defects in joint surfaces by neochondrogenesis with autogenous osteoperiosteal grafts stimulated by continuous passive motion. An experimental investigation in the rabbit. Clin. Orthop. Relat. Res., 208, 131140.

Pittenger, M.F., Mackay, A.M., Beck, S.C., Jaiswal, R.K., Douglas, R., Mosca, J.D., Moorman, M.A., Simonetti, D.W., Craig, S. \& Marshak, D.R. (1999) Multilineage potential of adult human mesenchymal stem cells. Science, 
284, 143-147.

Rubio, D., Garcia-Castro, J., Martín, M.C., de la Fuente, R., Cigudosa, J.C., Lloyd, A.C. \& Bernad, A. (2005) Spontaneous human adult stem cell transformation. Cancer Res., 65, 3035-3039.

Sakaguchi, Y., Sekiya, I., Yagishita, K. \& Muneta, T. (2005) Comparison of human stem cells derived from various mesenchymal tissues: superiority of synovium as a cell source. Arthritis Rheum., 52, 2521-2529.

Sohn, D.H., Lottman, L.M., Lum, L.Y., Kim, S.G., Pedowitz, R.A., Coutts, R.D. \& Sah, R.L. (2002) Effect of gravity on localization of chondrocytes implanted in cartilage defects. Clin. Orthop. Relat. Res., 394, 254-262.

Steadman, J.R., Rodkey, W.G. \& Rodrigo, J.J. (2001) Microfracture: surgical technique and rehabilitation to treat chondral defects. Clin. Orthop. Relat. Res., 391, Suppl. 362-369.

Strem, B.M., Hicok, K.C., Zhu, M., Wulur, I., Alfonso, Z., Schreiber, R.E., Fraser, J.K. \& Hedrick, M.H. (2005) Multipotential differentiation of adipose tissue-derived stem cells. Keio J. Med., 54, 132-141.

Wakitani, S., Goto, T., Pineda, S.J., Young, R.G., Mansour, J.M., Caplan, A.I. \& Goldberg, V.M. (1994) Mesenchymal cell-based repair of large, full-thickness defects of articular cartilage. J. Bone Joint Surg. Am., 76, 579-592.

Wickham, M.Q., Erickson, G.R., Gimble, J.M., Vail, T.P. \& Guilak, F. (2003) Multipotent stromal cells derived from the infrapatellar fat pad of the knee. Clin. Orthop. Relat. Res., 412, 196-212.

Xu, Y., Balooch, G., Chiou, M., Bekerman, E., Ritchie, R.O. \& Longaker, M.T. (2007) Analysis of the material properties of early chondrogenic differentiated adipose-derived storomal cells (ASC) using an in vitro three-dimensional micromass culture system. Biochem. Biophys. Res. Commun., 359, 311-316.

Yamamoto, N., Akamatsu, H., Hasegawa, S., Yamada, T., Nakata, S., Ohkuma, M., Miyachi, E., Marunouchi, T. \& Matsunaga, K. (2007) Isolation of multipotent stem cells from mouse adipose tissue. J. Dermatol. Sci., 48, 43-52.

Yamamoto, N., Majima, K. \& Marunouchi, T. (2008) A study of the proliferating activity in lens epithelium and the identification of tissue-type stem cells. Med. Mol. Morphol., 41, 83-91.

Yoshimura, K., Shigeura, T., Matsumoto, D., Sato, T., Takaki, Y., Aiba-Kojima, E., Sato, K., Inoue, K., Nagase, T., Koshima, I. \& Gonda, K. (2006) Characterization of freshly isolated and cultured cells derived from the fatty and fluid portions of liposuction aspirates. J. Cell. Physiol., 208, 64-76.

Zuk, P.A., Zhu, M., Mizuno, H., Huang, J., Futrell, J.W., Katz, A.J., Benhaim, P., Lorenz, H.P. \& Hedrick, M.H. (2001) Multilineage cells from human adipose tissue: implications for cell-based therapies. Tissue Eng., 7, 211-228. 